\title{
Stochastics and EUV Patterning in the 1x-nm Regime
}

\author{
Patrick Naulleau $^{1}$, Christopher Anderson ${ }^{1}$, Weilun Chao ${ }^{1}$, \\ Suchit Bhattarai ${ }^{2}$, and Andrew Neureuther ${ }^{2}$ \\ ${ }^{1}$ Center for X-ray Optics, Lawrence Berkeley National Laboratory, Berkeley, CA, USA \\ ${ }^{2}$ EECS, University of California, Berkeley, CA, USA
}

\begin{abstract}
The problem of stochastics in photoresist patterning is gaining increased attention. Understanding this problem requires new modeling methods. Here we describe the use of the Multivariate Poisson Propagation Model (MPPM) to study the relative importance of a variety of stochastic terms in both chemically amplified and non-chemically amplified resists. The results show that for the chemically amplified case, materials stochastic effects are on par with photon stochastics effects. The model is used to study both line-width roughness (LWR) impacts as well as contact size variations (CDU). As one might expect, contact CDU follows the same trends as LWR, but places even more sever constraints on the stochastic terms when considering the same feature size. Noting the contact patterning challenge, we also describe a phase-shift mask patterning method enabling the photon effect to be greatly reduced. At equivalent CDU, we demonstrate an effective $7 \mathrm{x}$ reduction in required source dose when patterning $25-\mathrm{nm}$ half pitch contacts.
\end{abstract}

Keywords: photoresist, extreme ultraviolet, stochastics, phase-shift mask

\section{Introduction}

The problem of stochastics in photoresist patterning is gaining increased attention. Modeling resist patterning stochastics is critical to understanding the practical implications of the effects, but requires new modeling methods [1-7]. Here we describe the use of the Multivariate Poisson Propagation Model (MPPM) $[2,4,7,8]$ to study the relative importance of a variety of stochastic terms in both chemically amplified (CA) and nonchemically amplified resists. We examine the impact of stochastics on both line-width roughness (LWR) and contact critical dimension uniformity (CDU).

Even assuming nearly ideal material conditions, photon stochastics have been shown to remain a significant concern, especially for contact CDU leading to relatively high dose requirements. These requirements, however, can be mitigated through the implementation of a checkerboard chromeless phase shift masks [9-12]. Using this method, a throughput gain of $8 \mathrm{X}$ has been demonstrated using an EUV aerial image microscope $[11,13]$. Here we demonstrate the method effectiveness using resist patterning results from the Berkeley Microfield Exposure Tool (MET) [14].

\section{Stochastic modeling}

To study the LWR and CDU impact of stochastic photon and material effects we use the MPPM. In this model, all relevant counting terms are treated as Poisson random variables instead of average values. Note that this is not the same as a Monte Carlo model and in principle can be treated as an analytic model. In the implementation used here [8], however, the model is implemented numerically. The modeling process begins with an arbitrary aerial image representing the time averaged photon density as a function of position (pixel). The first random variable represents the absorbed photons per pixel thus capturing the photon noise effect. The next random variable represents the number of chemical events per absorbed photon. This is a function of both the 
chemical yield, or quantum efficiency (QE), random variable as well as the local photoactive chemical density random variable. For the rest of the description below, we consider the case of a CA resist. In this case, the local photoactive chemical density is the photo-acid generator (PAG) density. The outcome of this step is a random variable representing the number of acids. The next step includes a reaction-diffusion process where there is an interaction with the local quencher concentration (also a random variable), as well as the protecting group concentration (yet another random variable). The end result is a random variable representing the deprotection ratio. Finally a threshold develop model is used to yield the stochastic pattern that can be analyzed using conventional LWR or CDU analysis software [15]

Each of the input random variables described above can be viewed as a counting problem. In each case the relevant value is the number of events or particles per unit volume. From the stochastic perspective, these cases are conveniently represented as Poisson random variables. The model then consists of a series of Poisson random variables acted upon and combined through a series of functions. Thus the problem can be viewed as a classical error propagation problem. Note that since the MPPM is implemented numerically, one is not restricted to using Poisson distributions; any desired distribution can be used for each of the random variables.

Although described above in terms of a CA resist, the model can also be applied to non-CA resists. In this case, the PAG concentration is replaced by the relevant photo-active element and the quencher is set to zero.

Examples of determinist variables used in the MPPM include the aerial image, absorptivity, electron mean free path, chemical diffusion range, quencher diffusion range, and chemical reaction rates. Also, not being a Monte Carlo model, each random variable can readily be substituted in the model with its mean value allowing the impact of individual stochastic terms to be assessed. Note that since the MPPM is essentially an error propagation model, deterministic variables will still have an impact on the stochastic output given that those variables affect the functions acting on the input and propagated random variables.

\section{Relative importance of stochastic terms}

As previously reported [11], the MPPM model has been shown to be a good predictor of both mean-field (non-stochastic) effects as well as overall stochastics. Here we use the model ability to isolate specific stochastic effects to study the relative importance of the various terms described above. We first consider a conventional polymer CA resist with a dose to size for $16-\mathrm{nm}$ lines space features of $33 \mathrm{~mJ} / \mathrm{cm}^{2}$. The patterning performance of this resist in the Berkeley MET tool [14] is shown in Fig. 1.

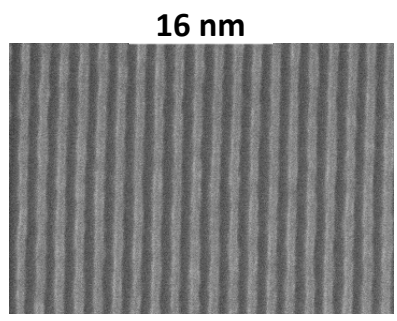

$14 \mathrm{~nm}$

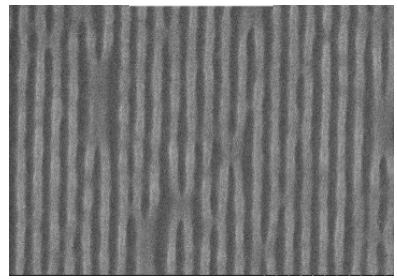

Fig. 1. Line space patterning results for a leading performance CA resist in the Berkeley MET tool.

Using supplier provided estimates for the material parameters, a resist thickness of 35 $\mathrm{nm}$, and estimating the acid diffusion based on the measured LWR correlation length [7], Table 1 shows the model predicted LWR and CDU (both $3 \sigma$ values) for $16-\mathrm{nm}$ dense features. For computation of the line-space aerial image, we use optimized dipole illumination and for the contacts we use optimized quadrupole illumination. The error bars are a result of the finite sample size used in the simulation.

The model shows that for this resist, the total material term (the root sum square of all the terms except for the photon term) to be greater than the photon term. Moreover, of the material terms, the quencher dominates. The quencher dominating is simply a result of that being the term of the smallest mean count therefore suffering from the largest relative stochastic variation. 
Table 1. MPPM predicted LWR and CDU terms for 16-nm dense features.

\begin{tabular}{lll}
\hline Stochastic terms & $\boldsymbol{L} \boldsymbol{W R}(\boldsymbol{n m})$ & $\boldsymbol{C D U}(\boldsymbol{n m})$ \\
\hline Photon & $2.0 \pm 2 \%$ & $3.0 \pm 8 \%$ \\
Acid & $1.2 \pm 2 \%$ & $1.9 \pm 8 \%$ \\
PAG & $0.6 \pm 2 \%$ & $1.1 \pm 8 \%$ \\
Quencher & $1.9 \pm 2 \%$ & $2.6 \pm 8 \%$ \\
Protecting groups & $0.1 \pm 2 \%$ & $0.1 \pm 8 \%$ \\
ALL & $3.0 \pm 2 \%$ & $4.1 \pm 8 \%$ \\
\hline
\end{tabular}

Fundamentally, the output variability, be it LWR or CDU is determined by the input noise (stochastic terms) and the functional coupling determined by the deterministic or mean-field terms. This means, for example, that the photon noise induced LWR as shown in Table 1 should not be viewed as an absolute limit of the counting terms, but rather it can also be modified by deterministic parameters in the model including both resist and system parameters. A simple example of this is to consider the aerial image contrast. For the linespace analysis above, which had assumed dipole illumination, the aerial image contrast was $85 \%$, if instead this contrast is reduced to $55 \%$, we get the results in Table 2 which shows a very significant increase in the coupling of all the input noise terms and raising the total LWR by $66 \%$.

Table 2. MPPM predicted LWR for 16-nm lines and spaces using identical resist parameters as in Table 1 , but reducing the aerial image contrast from $85 \%$ to $55 \%$.

\begin{tabular}{ll}
\hline Stochastic terms & LWR $(\boldsymbol{n m})$ \\
\hline Photon & $3.2 \pm 2 \%$ \\
Acid & $1.9 \pm 2 \%$ \\
PAG & $0.9 \pm 2 \%$ \\
Quencher & $3.1 \pm 2 \%$ \\
Protecting groups & $0.1 \pm 2 \%$ \\
ALL & $4.9 \pm 2 \%$ \\
\hline
\end{tabular}

The results above show absorbed photon and quencher counts to be the dominant input noise contributors to LWR and CDU for a conventional CA resist. Both these terms, however, can be mitigated by considering inorganic non-CA resists. The ability to include metal in significant quantities allows absorption to be increased substantially, thus for the same exposure dose many more photons contribute to the pattern thereby reducing the photon noise. A typical polymer resist will have an absorptivity of approximately $4 \mu \mathrm{m}^{-1}$ whereas metal oxide resists have been shown to achieve values as high as $20 \mu \mathrm{m}^{-1}$ [16]. Note, however, that there are limits to the acceptable absorptivity since making it too high could cause exposure problems through thickness and thus sidewall slope issues. At a value of 20 $\mu \mathrm{m}^{-1}$, a $20-\mathrm{nm}$ thick resist will have $33 \%$ less dose at the bottom of the resist compared to the top.

Another benefit of the non-CA resist is that by eliminating reliance on quencher, the nonCA resist can improve chemical/material stochastics as well. Table 3 shows the model predicted LWR and CDU terms for 16-nm dense features for an assumed non-CA resist. The same aerial image parameters as used in Table 1 are used here. The model parameters include: absorptivity $=20 \mu \mathrm{m}^{-1}$, thickness $=20$ $\mathrm{nm}$, sensitivity $=60 \mathrm{~mJ} / \mathrm{cm}^{2}$, quantum efficiency (radiation chemistry events per absorbed photon $)=2$, chemical blur $=12 \mathrm{~nm}$, and the active chemical component density $=$ $2 / \mathrm{nm}^{3}$. The patterning performance of a of nonCA resist expected to have properties similar to those described above is shown in Fig. 2.

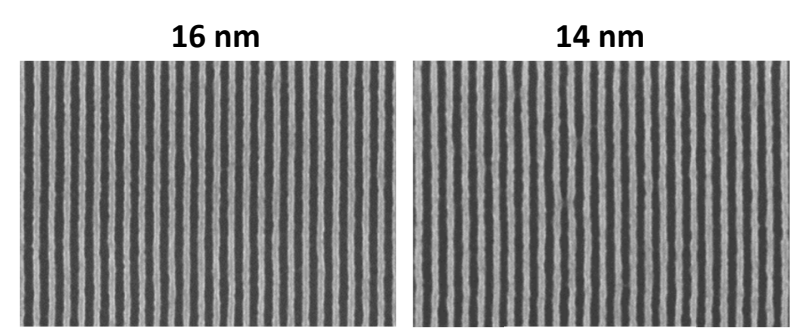

$13 \mathrm{~nm}$

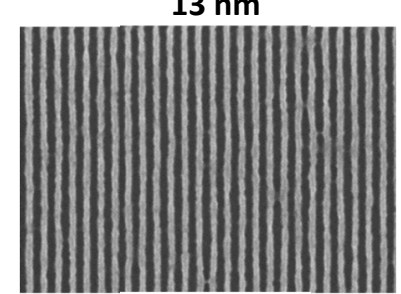

Fig. 2. Line space patterning results for a leading performance non-CA resist in the Berkeley MET tool. 
Table 3. MPPM predicted LWR and CDU terms for 16$\mathrm{nm}$ dense features in an assumed non-CA resist.

\begin{tabular}{lll}
\hline Stochastic terms & LWR (nm) & $\boldsymbol{C D U}(\mathbf{n m})$ \\
\hline Photon & $1.2 \pm 2 \%$ & $1.5 \pm 8 \%$ \\
Photochemistry & $0.9 \pm 2 \%$ & $1.0 \pm 8 \%$ \\
Active components & $0.2 \pm 2 \%$ & $0.3 \pm 8 \%$ \\
ALL & $1.6 \pm 2 \%$ & $1.8 \pm 8 \%$ \\
\hline
\end{tabular}

\section{Contact CDU projections}

Assuming a contact CDU requirement of $10 \%$ of $\mathrm{CD}$, we see that even the model nonCA resist from Table 3 does not meet the requirements. If we instead assume a sensitivity of $80 \mathrm{~mJ} / \mathrm{cm}^{2}$ and a chemical blur of $7 \mathrm{~nm}$ (a parameter that has been demonstrated experimentally [11]), while keeping the other parameters as described for Table 3, the MPPM shows that a CDU of $1.2 \mathrm{~nm}$ can be achieved. Figure 3 shows the MPPM output.

While the result in Fig. 3 outperforms the $10 \%$ requirement, it provides headroom for considering even smaller contacts. Table 4 shows the MPPM predicted contact CDU as a function of half pitch while keeping all the resist parameters identical to those described for Fig. 3 and directly scaling the aerial image thereby assuming that the optical performance is improving. Note that although some of the relative changes appear to be on the order of the reported error bars, the model ability to predict relative performance is much superior to the reported precision which is a function of the dataset size ( 81 contacts in this case). The model, however, has the ability to use a predefined random number generator seed allowing the stochastic distributions to be replicated from run to run. For example, Fig. 4 shows the MPPM results for $10 \mathrm{~nm}$ contacts where one can observe the same relative distribution of contact sizes as compared to the 16-nm case in Fig. 3. Therefore, the relative performance will not suffer from the same dataset size limited uncertainty as do the individual datasets.

The results in Table 4 show that once the CD drops to below $14 \mathrm{~nm}$, the $10 \% \mathrm{CDU}$ requirement can no longer be met for the assumed resist and aerial image parameters. We thus next consider which parameters would allow the $10 \% \mathrm{CDU}$ requirement to be met even at 10 half pitch without increasing the dose. Noting that the quantum efficiency (QE) plays a key role in the chemical noise term and that the resist blur is important to the coupling of stochastic noise to final CDU, we consider increasing the QE from 2 to 4 and decreasing the blur from $7 \mathrm{~nm}$ to $4 \mathrm{~nm}$. Doing so, we can achieve a CDU of $1 \mathrm{~nm}$ on $10-\mathrm{nm}$ contacts as shown in Fig. 5.

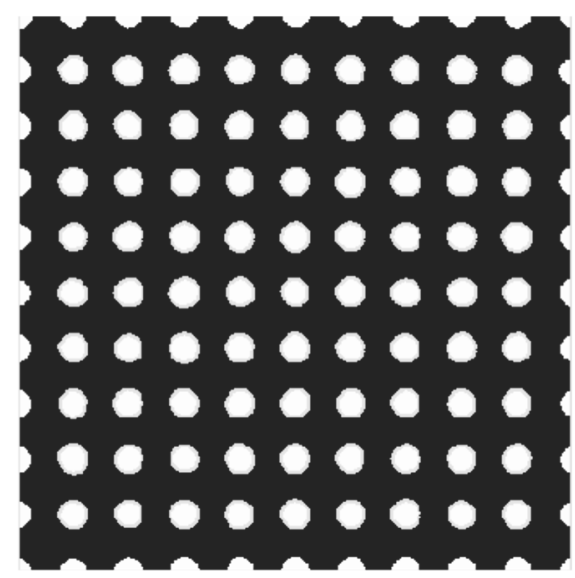

Fig. 3. MPPM output for 16-nm dense contacts and non-CA parameters. Results show a CDU of $1.2 \mathrm{~nm}$.

Table 4. MPPM predicted contact CDU as a function of contact half pitch.

\begin{tabular}{ll}
\hline Half pitch $(\mathbf{n m})$ & $\boldsymbol{C D U}(\mathbf{n m})$ \\
\hline 16 & $1.2 \pm 8 \%$ \\
14 & $1.3 \pm 8 \%$ \\
12 & $1.5 \pm 8 \%$ \\
10 & $1.7 \pm 8 \%$ \\
\hline
\end{tabular}

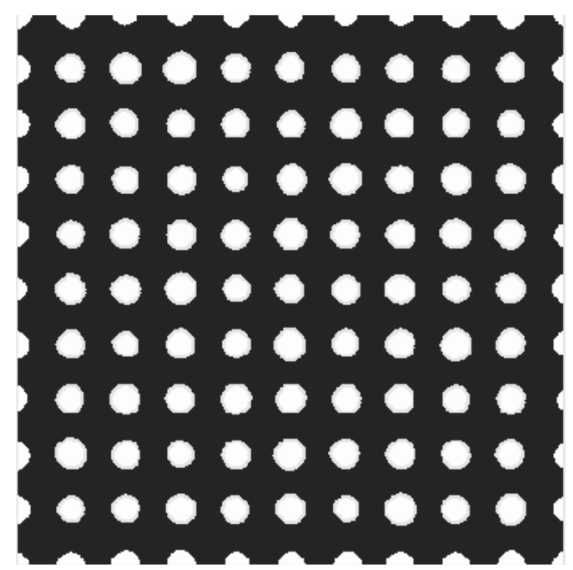

Fig. 4. MPPM output for 10-nm dense contacts. 


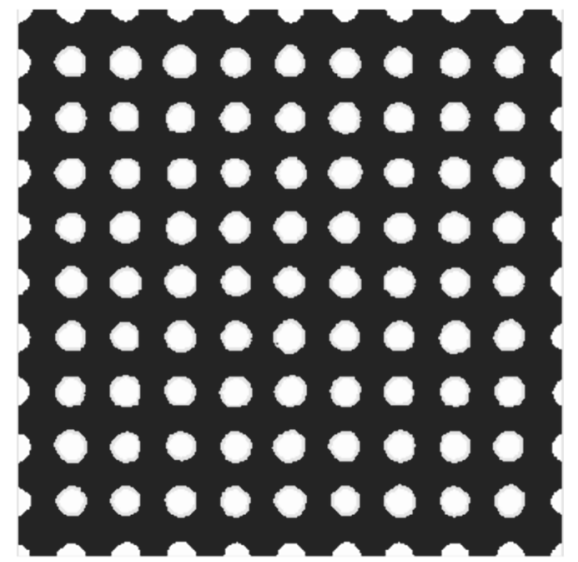

Fig. 5. MPPM output for $10-\mathrm{nm}$ dense contacts after increasing QE to 4 and decreasing resist blur to $4 \mathrm{~nm}$.

Although Fig. 5 shows that $10 \% \mathrm{CDU}$ is achieved at $10-\mathrm{nm}$ with the assumed resist parameters, the dose in this case is still 80 $\mathrm{mJ} / \mathrm{cm}^{2}$. Ideally one would like to reduce this dose. If we make the further assumption of increasing the absorptivity to $30 \mu^{-1}$ and further decreasing the blur to $3 \mathrm{~nm}$, we can achieve a CDU of $1 \mathrm{~nm}$ at a dose of $50 \mathrm{~mJ} / \mathrm{cm}^{2}$. Note, however, that at this level of absorptivity, we will have nearly $50 \%$ less dose at the bottom of the $20-\mathrm{nm}$ resist film as compared to the top.

\section{Reducing contact dose requirements}

The results above suggest that it will be extremely challenging to achieve high sensitivity resists as we extend EUV towards the single digit nm regime. Dose requirements for contacts, however, can be mitigated through the implementation of a checkerboard chromeless phase shift masks [9-12]. EUV aerial image microscopy [11] has been used to report on the feasibility of this method for dose reduction. Demonstration of the method has now been extended to lithographic patterning using the Berkeley MET. Figure 6 shows 25$\mathrm{nm}$ contacts in a CA resist directly comparing a conventional absorber mask and an etched multilayer phase shift mask. The phase shift mask is seen to yield a $7 \mathrm{x}$ effective sensitivity gain. Note that the actual dose at the wafer is not actually changed, as must be the case since we are using the same resist. What is changed is the optical efficiency of the mask. This is important since if the resist were truly being patterned with $7 \mathrm{x}$ fewer photons at the resist, the stochastics would certainly suffer.

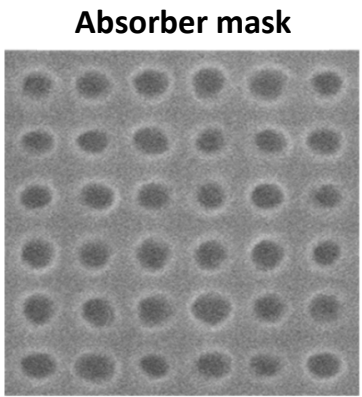

$94 \mathrm{~mJ} / \mathrm{cm}^{2}$
Phase shift mask

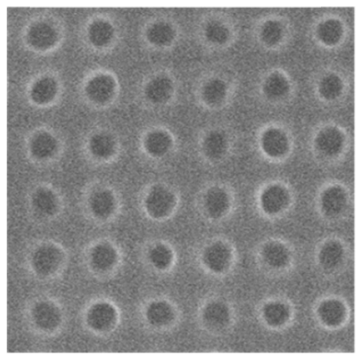

$13 \mathrm{~mJ} / \mathrm{cm}^{2}$
Fig. 6. Direct comparison of the effective patterning dose for 25-nm contacts in the Berkeley MET for conventional absorber mask and etched multilayer phase shift mask.

\section{Summary}

The MPPM model has been used to show that in the case of current CA resists, materials and photon stochastics are equally important. Key to improvement is to increase both the number of photons contributing to the pattern and the chemical density. Increasing the photons can come from decreased sensitivity (not ideal) or increased absorptivity. By far the limiting term for chemical density is the quencher in CA resists. This could be addressed by increasing quencher concentration or changing the resist platform to non-CA.

The results suggest that it will be extremely challenging to achieve high sensitivity resists as we extend EUV towards the single digit $\mathrm{nm}$ regime, especially for contacts. This problem, however, could be mitigated through the implementation of phase shift masks. Here we have used the Berkeley MET to demonstrate the method achieving an effective patterning dose reduction of $7 x$.

The authors are greatly indebted to the CXRO MET operations team including Warren Holcomb, Gideon Jones, Chanin King, and Jessalyn Sincher. We also thank Rama Ayothi of JSR, Hideaki Tsubaki of Fuji, and Jason Stowers of Inpria for excellent resist support. We also acknowledge equipment support from Intel, Samsung, TSMC, Inpria, and JSR. The work was performed at Lawrence Berkeley National Laboratory's Advanced Light Source synchrotron facility with industry funding through the U.S. Department of Energy under Contract No. DE-AC02-05CH11231. 


\section{References}

1. G. Gallatin and J. A. Liddle, Microelectr. Eng., 46 (1999) 365.

2. C. Anderson and P. Naulleau, J. Vac. Sci. Technol. B, 27 (2009) 665.

3. C. Mack, J. Vac. Sci. Technol. B, 27 (2009) 1122.

4. P. Naulleau and G. Gallatin, J. Vac. Sci. Technol. $B, 28$ (2010) 1259.

5. J. Biafore and M. Smith, Proc. SPIE, $\mathbf{8 3 2 5}$ (2012) $83250 \mathrm{H}$.

6. T. Kozawa, J. Santillan, and T. Itani, Appl. Phys. Express, 6 (2013) 026502.

7. P. Naulleau, C. Anderson, W. Chao, S. Bhattarai, and A. Neureuther, J. Photopolym. Sci. Technol., 27 (2014) 747.

8. Stochastic modeling performed using SuMMIT software (www.lithometrix.com).

9. K. Toh, G. Dao, R. Singh, and H. Gaw, Proc. SPIE, 1496 (1991) 27.

10. P. Naulleau, C. Anderson, W. Chao, S. Bhattarai, A. Neureuther, K. Cummings, S. Jen, M. Neisser, and B. Thomas, J. Photopolym. Sci. Technol., 27 (2014) 725.
11. P. Naulleau, C. Anderson, W. Chao, K. Goldberg, A. Wojdyla, S. Bhattarai, A. Neureuther, F. Goodwin, and M. Neisser, $J$. Photopolym. Sci. Technol., 28 (2015) 777.

12. P. Naulleau, C. Anderson, W. Chao, K. Goldberg, E. Gullikson, F. Salmassi, and A. Wojdyla, Proc. SPIE, 9984 (2016) 99840P.

13. K. Goldberg, M. Benk, A. Wojdyla, P. Naulleau, W. Chao, D. Johnson, A. Donoghue, R. Miyakawa, W. Wang, and J. Macdougall, Proc. SPIE, 9422 (2015) 94221A.

14. P. Naulleau, K. A. Goldberg, E. H. Anderson, K. Bradley, R. Delano, P. Denham, B. Gunion, B. Harteneck, B. Hoef, H. Huang, K. Jackson, G. Jones, D. Kemp, J. A. Liddle, R. Oort, A. Rawlins, S. Rekawa, F. Salmassi, R. Tackaberry, C. Chung, L. Hale, D. Phillion, G. Sommargren, and J. Taylor, Proc. SPIE, 5374 (2004) 881.

15. LWR and CDU analysis performed using SuMMIT software (www.lithometrix.com).

16. R. Fallica, J. Stowers, A. Grenville, A. Frommhold, A. Robinson, and Y. Ekinci, Proc. SPIE, 9776 (2016) 977612. 